\title{
Visual Receptive Fields in the Striate-Recipient Zone of the Lateral Posterior-Pulvinar Complex
}

\author{
Leo M. Chalupa and Bruce P. Abramson ${ }^{2}$ \\ Department of Psychology and the Physiology Graduate Group, University of California, Davis, California 95616
}

The lateral posterior (LP)-pulvinar complex of the cat is known to contain multiple visual areas. In the present study, we examined the receptive field properties of single neurons isolated in the lateral division of this complex (the LPI). The LPI is designated the striate-recipient zone because it is the only region of the LP-pulvinar receiving cortical projections from areas 17 and 18 . The recordings revealed that the striate-recipient zone of LP comprises 2 subareas, which we have termed LPI-1 and LPI-2. In the main segment (LPI-1), virtually all cells responded securely to visual stimuli. The vast majority of these neurons were binocular, with relatively small and well-defined receptive fields. More than half of the cells were found to be directionally selective, and almost this many were orientation specific. The orientation tuning of these cells was found to be quite precise, comparable to complex cells in area 17. In contrast, in the small dorsolateral segment of the striate-recipient zone (the LPI-2), a substantial proportion of cells could not be visually activated. Here, the visual cells had very large receptive fields, and relatively few were direction or orientation selective. The LPI-2 receives subcortical inputs from the superficial layers of the superior colliculus, the hypothalamus, and cerebellum, while the LPI- 1 is innervated only by cortical axons. It is suggested that the subcortical connections of the LPI-2 account for the differences in the response properties of the 2 striate-recipient areas.

The present results, in conjunction with our previous findings on the principal tectorecipient zone (Chalupa et al., 1983), permit 2 generalizations regarding the functional organization of the cat's LP-pulvinar complex. First, there are clear differences among the visual areas of the LP-pulvinar in the cellular processing of visual information. Second, these functional differences can be related to the principal sources of visual input to the various divisions of the LP-pulvinar.

The lateral posterior (LP)-pulvinar complex has long been implicated in visual function (Chalupa, 1977). In the cat, electrophysiological mapping experiments and anatomical tracing studies have indicated that there are at least 3 visual areas within the LP-pulvinar. The pulvinar proper contains a complete representation of the contralateral visual field, while the nucleus

\footnotetext{
Received Apr. 20, 1988; revised June 20, 1988; accepted June 21, 1988.

This work was supported by Grant BNS 84-00807 from the National Science Foundation.

Correspondence should be addressed to Leo M. Chalupa at the above address.

a Present address: School of Optometry, University of California, Berkeley, CA 94720.

Copyright (C) 1989 Society for Neuroscience $0270-6474 / 89 / 010347-11 \$ 02.00 / 0$
}

lateralis postcrior contains 2 such reprcscntations, one in the medial and the other in the lateral division (Mason, 1978; Raczkowski and Rosenquist, 1981; Hutchins and Updyke, 1984).

Each retinotopically defined area is characterized by a distinct set of projections from visual cortical and subcortical structures. The pulvinar is distinguished by a projection from the pretectum (Berman, 1977; Itoh, 1977; Graybiel and Berson, 1980; Weber et al., 1986), as well as cortical areas 19, 20a, and $21 \mathrm{a}$ (Kawamura et al., 1974; Updyke, 1977; Symonds et al., 1981). The medial division of LP (LPm) is innervated by neurons of the stratum griseum superficiale of the superior colliculus (Kawamura, 1974; Kawamura and Kobayashi, 1975; Graham, 1977; Graybiel and Berson, 1980; Caldwell and Mize, 1981; Raczkowski and Rosenquist, 1983; Abramson and Chalupa, 1988), and by cortical areas 19,21a, and the suprasylvian visual areas (Updyke, 1977; Raczkowski and Rosenquist, 1983). The lateral zone of the LP (LPI) is designated the striate-recipient region because it is the only division of the LP-pulvinar complex that receives inputs from areas 17 and 18 . The LPl is also innervated by neurons of areas $19,20,21$, and the suprasylvian and splenial visual cortical areas (Kawamura, 1974; Updyke, 1977, 1981; Symonds et al., 1981; Berson and Graybiel, 1983; Abramson and Chalupa, 1985a). The projections from areas 17 and 18 are derived only from neurons situated in layer 5 , whereas in all other visual cortical areas neurons in both layer 5 and 6 project to the I.P-pulvinar (Abramson and Chalupa, 1985a).

In contrast to the substantial amount of anatomical information that has accumulated over the last decade, there are still major gaps in our knowledge about the functional organization of the visual areas in the LP-pulvinar. While a number of studies have dealt with visual receptive field properties of LP-pulvinar neurons (Godfraind et al., 1972; Chalupa and Fish, 1978; Mason, 1978, 1981; Fish and Chalupa, 1979), much of this work was carried out before it was realized that this region of the thalamus contains multiple visual areas. As a consequence, little is yet known about how visual responses of single neurons in the different areas of the LP-pulvinar complex relate to their principal sources of visual input.

Several years ago we addressed this issue by comparing the receptive field properties of neurons in the principal tectorecipient zone (the LPm) with those of cells in the superficial layers of the superior colliculus (Chalupa et al., 1983). This study revealed that many visual response properties of LPm cells are strikingly similar to those of superior collicular neurons. However, it also became apparent that there are some clear differences in the way that information is processed by these 2 subcorlical components of the visual system.

In the present study, we have extended this approach to an 

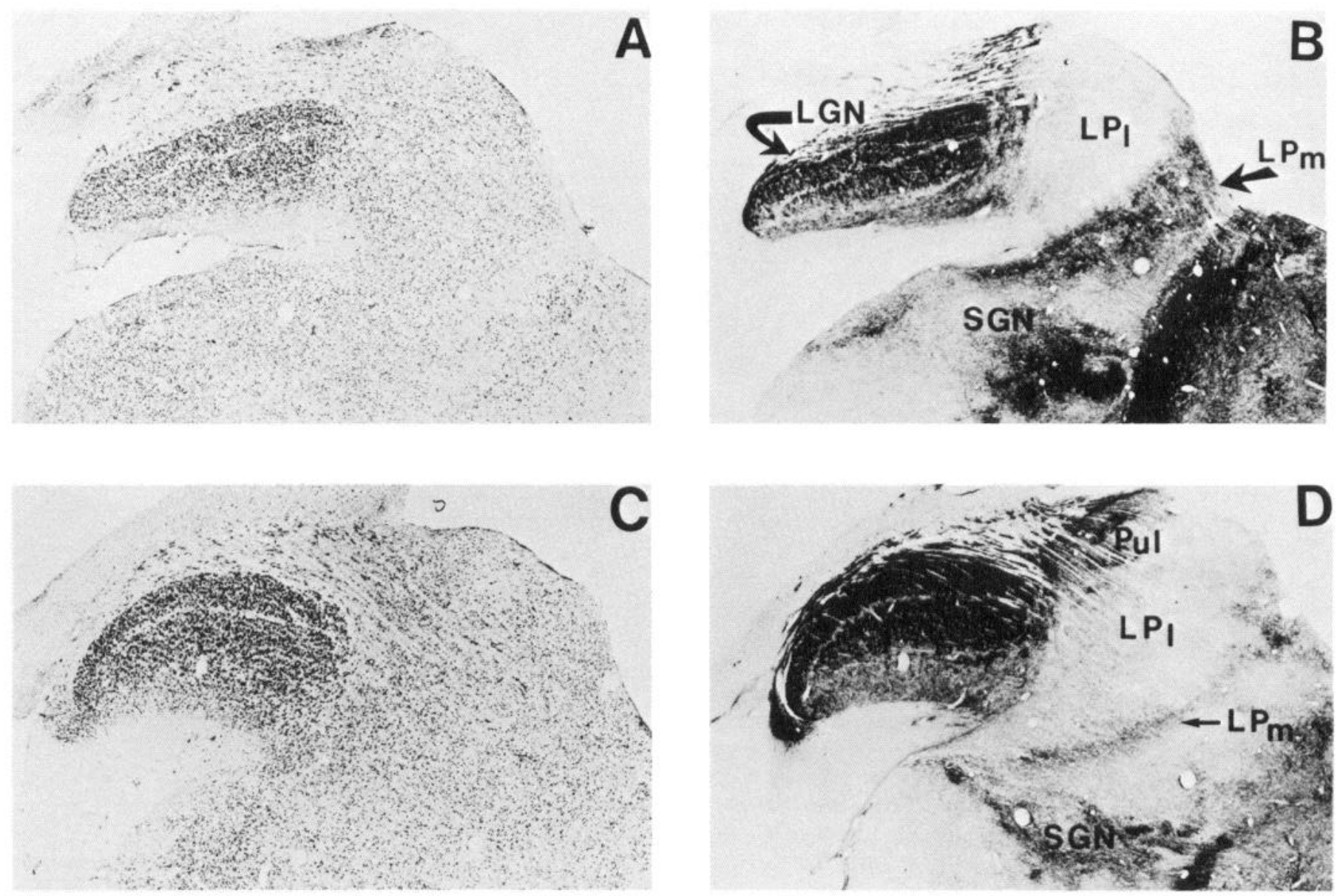

Figure 1. Photomicrographs of coronal sections through the posterior thalamus. The sections at the left $(A$ and $C)$ were stained with cresyl violet. Adjacent sections to the right $(B$ and $D)$ were stained for acetylthiocholinesterase. $A$ and $B$ were taken at the Horsley-Clarke frontal coordinate of approximately A7.5, whereas $B$ and $C$ were approximately $2 \mathrm{~mm}$ caudal. $L P m$, nucleus lateralis posterior, pars medialis; $L P l$, nucleus lateralis posterior, pars lateralis; $P u l$, pulvinar; $L G N$, lateral geniculate body; $S G N$, suprageniculate; $L D$, nucleus lateralis dorsalis.

investigation of the striate-recipient zone of the LP-pulvinar complex. Specifically, we documented the receptive field properties of LPl cells with 2 main objectives in mind. First, we sought to determine how the functional organization of the striaterecipient zone differs from that of the principal tectorecipient region. Second, we wanted to know if the visual responses of LPl cells can be related to the salient functional properties of the cortical neurons that project to this division of the cat's LPpulvinar complex.

\section{Materials and Methods}

Preparation. Experiments were conducted on 18 adult anesthetized cats. Each animal was premedicated with atropine $(0.08 \mathrm{mg} / \mathrm{kg})$ and ketamine hydrobromide $(15 \mathrm{mg} / \mathrm{kg})$. Following cannulation of the saphenous vein and tracheotomy, the animal was placed in a stereotaxic instrument adapted for visual neurophysiology. Heart rate was monitored continuously thereafter, and body temperature was maintained at $37^{\circ} \mathrm{C}$ by means of an electrical feedback control system. A small opening was made in the cranium over the LP-pulvinar complex, and 2 stainless steel screws were embedded bilaterally in the frontal sinus to serve as electrodes for monitoring EEG activity. A head-mount apparatus, cemented to the anterior portion of the skull with acrylic, obviated the use of eye, ear, and mouth bars.

The dura was reflected and the cortex covered with a mixture of mineral oil and petroleum jelly. The pupils were dilated, and the nictitating membranes were retracted with single drops of $1.0 \%$ atropine sulfate and $10 \%$ phenylephrine hydrochloride, respectively. Clear plastic lenses were fitted over the corneas. Animals were immobilized with gallamine triethiodide infused at a rate of $10 \mathrm{mg} / \mathrm{kg} / \mathrm{hr}$ and ventilated with a mixture of $70 \%$ nitrous oxide and $30 \%$ oxygen or with room air. End-tidal $\mathrm{CO}_{2}$ levels were continuously monitored with a Beckman LB-2 gas analyzer and maintained between 3.5 and $4.5 \%$. Anesthesia was maintained by intravenous injections of chloralose $(20 \mathrm{mg} / \mathrm{kg})$ about 1 $\mathrm{hr}$ before recordings were begun and with repeated injections at this dosage in approximately $4 \mathrm{hr}$ intervals throughout the recording session. This anesthetic state resulted in a cortical EEG pattern consisting of slow-wave activity with intermittent spindle bursts.

Recordings and visual stimulation. The optic disks were projected and plotted on a tangent screen situated $57 \mathrm{~cm}$ in front of the animal's eyes. The luminance of the tangent screen was $0.16 \mathrm{~cd} / \mathrm{m}^{2}$, and visual stimuli were $1.9 \log$ units above or below this background level. When necessary the eyes were refracted with supplementary lenses.

Extracellular recordings were made with varnish-insulated tungsten microelectrodes with an impedance of about $5 \mathrm{M} \Omega$ measured at $1 \mathrm{kHz}$. Activity was amplified with a Grass P15 or a Bak A-1 preamplifier, displayed on a Tecktronix 5111 storage oscilloscope and monitored through a Grass AM8 audio monitor. A window discriminator (WPI model 120) was used to feed standardized pulses to a computer for online analysis of the visual responses. Data were collected only for those units having biphasic potentials with a minimum waveform duration of $1 \mathrm{msec}$.

A variety of moving and stationary stimuli were employed while the microelectrode was slowly advanced into the LP-pulvinar. When a visually responsive cell was encountered, the activating region of the receptive field was plotted by presenting a flashing stationary or moving stimulus well outside the responsive area while gradually approaching its center. Typically, the smallest stimulus that elicited reliable responses 

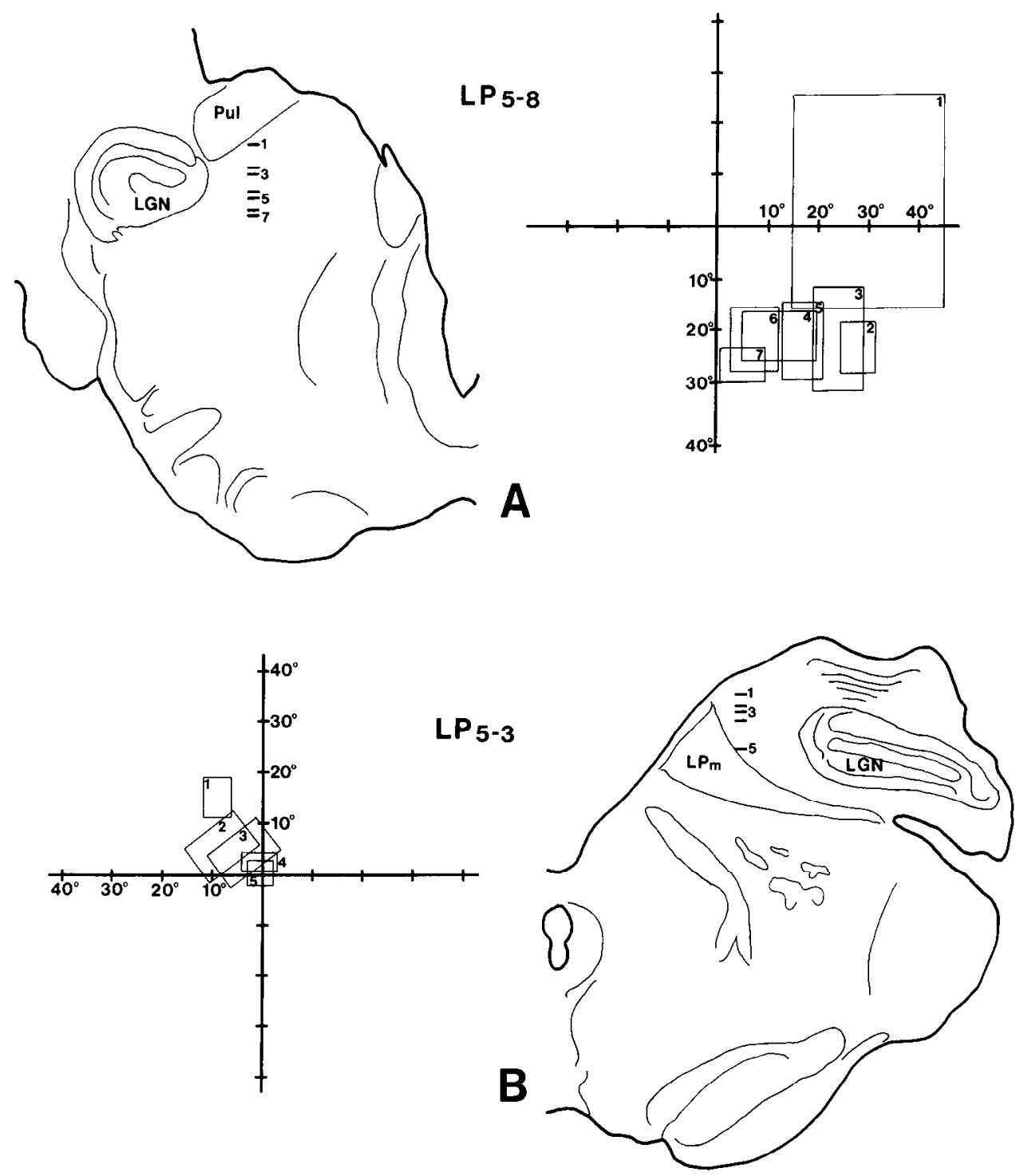

Figure 2. Location of visual receptive fields of cells isolated along penctrations through the LPl in 2 animals [7 cells from the animal designated $\mathrm{LP}_{5-8}$ $(A)$ and 5 cells from $\left.\mathrm{LP}_{\mathrm{s}-3}(B)\right]$. The section in $A$ was taken from a level representing a Horsley-Clarke frontal coordinate of approximately A7.5; $B$, A5.5. The first receptive field in each penetration was plotted for a neuron isolated in the most dorsal portion of the track. The numbers associated with each receptive field plot correspond to the similarly numbered hatch mark on the line drawing of the coronal section. The vertical and horizontal meridians are indicated by the heavy lines. Note the large receptive field from the dorsalmost cell in $A$. The region from which this cell was isolated contains cells with atypical response properties and is outlined in Figure 3.

was used for this purpose. The borders between responsive and unresponsive regions were drawn directly on a sheet of translucent paper mounted on the tangent screen. Each receptive field was referenced within the appropriate visual hemifield to the area centralis representation (Bishop et al., 1962).

For each cell we sought to determine the following response characteristics: ocular dominance, directional selectivity, orientation specificity, the internal organization of the receptive field, the presence of surround suppression, and the cut-off velocity to stimuli moved rapidly across the receptive field. The procedures employed to examine these response properties have been described previously (Chalupa et al., 1983). For most cells, poststimulus time histograms were performed on-line to document neuronal responses, but in many cases discharge patterns were assessed by listening to the audio monitor.

Several electrode penetrations were made through the LP nucleus of each animal and small electrolytic lesions $(5 \mu \mathrm{A}$ for $5 \mathrm{sec})$ were made along each track. At the end of the experiment the animal was administered a lethal dose of pentobarbital and perfused transcardially with a solution of $0.9 \%$ sodium chloride and $1.0 \%$ heparin sulfate. This was immediately followed by a solution of $10 \%$ formalin buffered with 0.1 M phosphate. All solutions were kept at $\mathrm{pH} 7.4$ with $0.1 \mathrm{M}$ phosphate buffer. The appropriate portion of the brain was blocked in situ and stored overnight in buffered sucrose solution at $4^{\circ} \mathrm{C}$. Coronal sections were cut frozen at $50 \mu \mathrm{m}$ on a sliding microtome.

Histochemistry. In Nissl-stained sections differentiating the LPl from adjacent regions of the LP-pulvinar complex is problematic. Accordingly, as in previous studies (Chalupa et al., 1983; Abramson and Cha- lupa, 1985a, 1988), we used a cholinesterase histochemical method to facilitate differentiation of the $\mathrm{LPl}$ region. A modification of the direct coloring methods of Karnovsky and Roots (1964) and Butcher et al (1974) was used to demonstrate cholinesterase activity. A detailed description of this method has been provided elsewhere (Abramson and Chalupa, 1988).

Verification of recording sites. Small identifying lesions are often difficult to identify in tissue processed for cholinesterase histochemistry. Therefore, alternate sections through the LP nucleus were treated to demonstrate cholinesterase activity or stained for Nissl substance with cresyl violet. Electrode penetrations were reconstructed by drawing the cresyl violet-stained sections showing the marking lesions. The major divisions of the LP-pulvinar complex were then traced onto these drawings from adjacent cholinesterase-stained sections.

\section{Results}

The results are based on recordings from 279 cells isolated in the striate-recipient region of the LP-pulvinar. We relied upon cholinesterase histochemistry to define the LPl in penetrations made through the caudal portion of the thalamus. As depicted in Figure 1, in caudal sections the LPl stained lightly so that its border can be clearly differentiated from the adjacent LPm (the principal tectorecipient region), which is darkly stained by the cholinesterase procedure. At more rostral levels, the LPm is no 
Figure 3. Loci of the 279 cells whose response properties were examined in this study are plotted as dots on tracings of representative coronal sections stained with acetylthiocholinesterase. Numbers below each section refer to the corresponding Horslcy-Clarke frontal coordinate. The shaded portion represents the region of the LPI in which the cells with large receptive fields and relatively nonspecific response characteristics were isolated. Abbreviations are the same as for Figure 1.
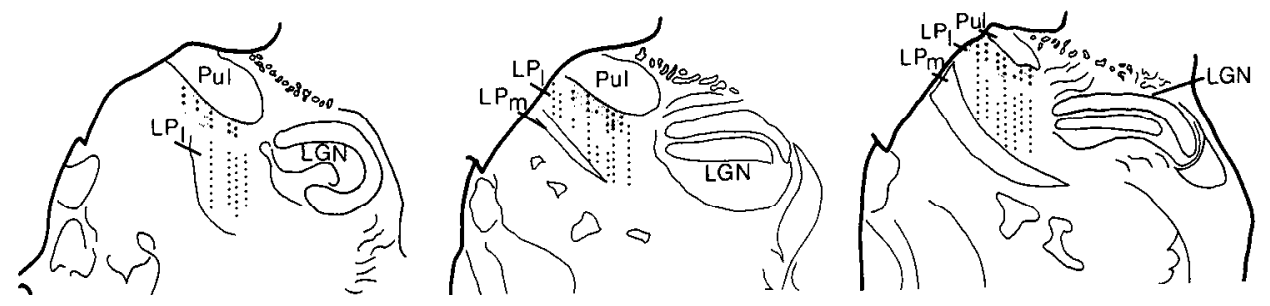

7.5

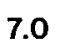

6.5

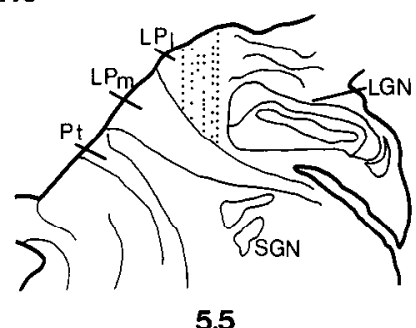

$\mathrm{N}=\mathbf{2 7 9}$ longer present, and the histochemical stain cannot, therefore, be used to delineate the ventral and medial boundaries of the striate-recipient zone. However, in some rostral penetrations the retinotopic organization of the LPI enabled us to identify the striate-recipient zone. In these cases, as the electrode was advanced towards the ventromedial border of the LPI, the position of receptive fields plotted for successive cells shifted progressively towards the vertical meridian (see Fig. 2). If neither the retinotopic organization nor the histochemistry proved adequate to assign cells with confidence to the LPl, the data were discarded from further analysis.

Even in initial experiments, it became apparent that the visual responsivity of cells localized near the border of the pulvinar (that is, in the dorsolateral segment of the LPl) differed markedly from those isolated in the remainder of the striate-recipient region. This zone extended about $0.2-0.4 \mathrm{~mm}$ into the $\mathrm{LPl}$ along the boundary with the pulvinar. We will consider the functional organization of this area after describing the visual responses in the main segment of the LPl. The loci of the cells we studied in the 2 subareas of the LPl are illustrated in Figure 3.

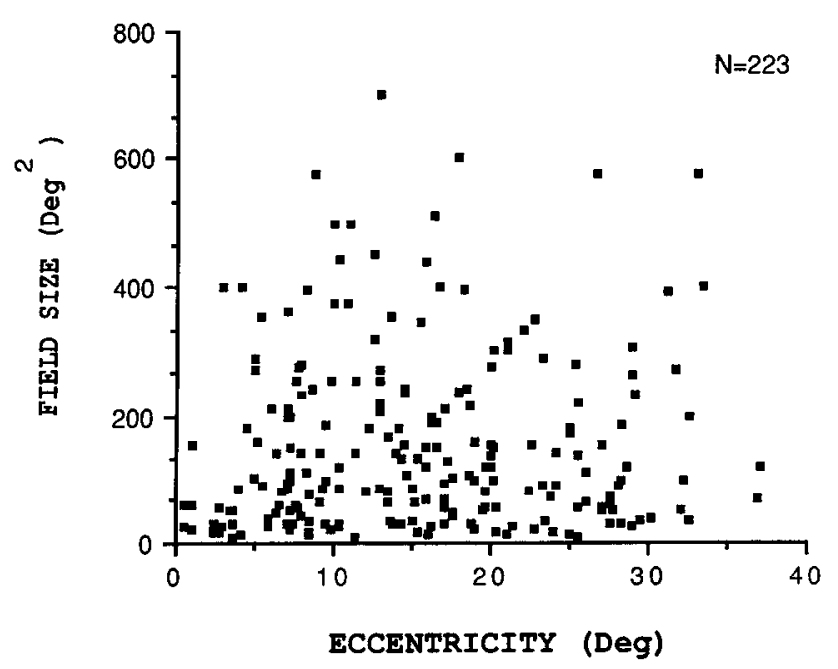

Figure 4. Receptive field area as a function of eccentricity (distance of receptive field center from the area centralis representation) for 223 cells in the LPI. The correlation between receptive field size and eccentricity is obviously not significant.
In the main segment of the LPl virtually all cells responded securely to visual activation $(97 \%, 224$ of 232$)$. The receptive fields of all these neurons were within $40^{\circ}$ of the area centralis representation, in the binocular portion of the visual field. Our electrode penetrations sampled from widespread regions of the LPl (see Fig. 3) without encountering cells with more peripheral receptive field centers. It is also noteworthy that the receptive fields of many cells crossed the vertical meridian, in some instances by as much as $20^{\circ}$.

As may be seen in Figure 4, the size of the receptive fields varied considerably at any given eccentricity. Further, other than within $5^{\circ}$ of the area centralis representation, where almost all cells had relatively small receptive fields, the size of the fields did not vary appreciably with changes in eccentricity. It should be noted that our measurements included only the activating region of the field, the region in the visual field from which reliable discharges could be elicited. Many of these receptive fields were flanked by "silent" suppressive surrounds (see below), but we did not attempt to delimit the extent of thesc suppressive regions.

\section{Ocular dominance}

The degree of binocularity was assessed using the ocular dominance scale of Hubel and Wiesel (1962). As shown in Figure 5 , most cells were binocular, and the greatest number responded equally well to stimulation of either eye (group 4). Included in this group is a substantial number of neurons that exhibited clear binocular facilitation. For these neurons monocular stimulation of either eye alone was much less effective in activating the cell than binocular stimulation. In extreme cases, binocular stimulation was required to elicit secure responses. Of the monocular cells, almost all were driven by the contralateral eye (group 1). There was no apparent regional variation of ocular dominance classes within the LPl.

\section{Responses to movement}

With few exceptions LPl neurons responded reliably to both moving as well as stationary flashed stimuli (either spots or bars) that were smaller than the activating region of the receptive field. Clcar preferences for cither moving or stationary stimuli were rare, but we did not attempt to document subtle differences 


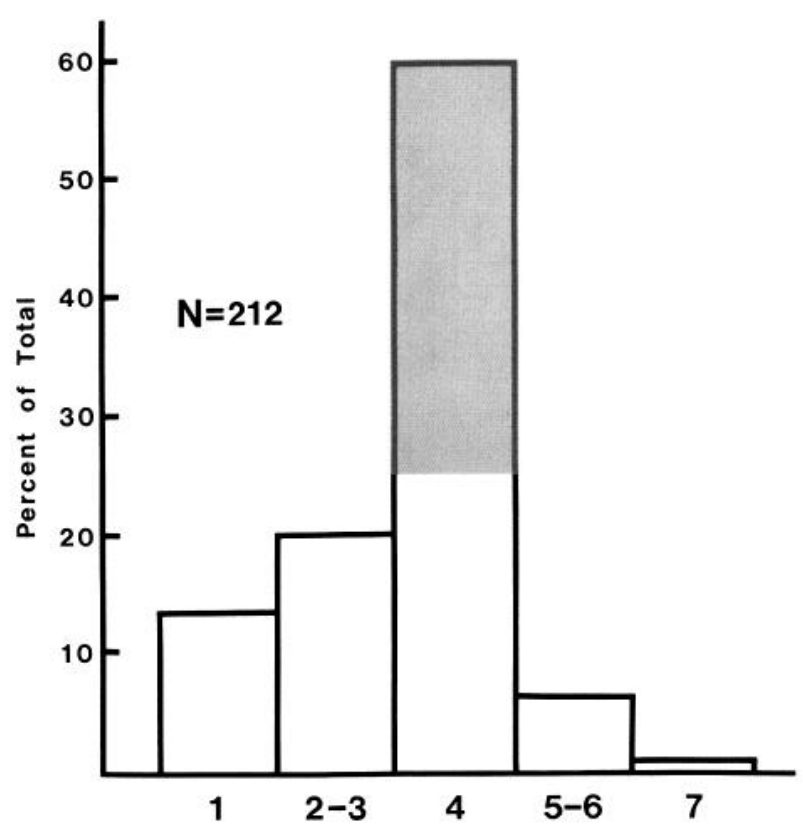

Figure 5. Ocular dominance of 212 cells in the LPl-1. Cells in group 1 responded to stimulation of the contralateral eye and those of group 7 could be activated only by the ipsilateral eye. Group 4 cells responded equally well to stimulation of either eye. The shaded portion in group 4 represents the proportion of cells that could be activated only by binocular stimulation or that responded better to binocular than monocular stimulation.

on this dimension. The stimulus speeds that were effective in activating these neurons spanned a broad range, and a sizable proportion (125/164) continued to respond reliably to stimulus speeds greater than $200 \% \mathrm{sec}$.

About $58 \%$ of the cells (129/221) were directionally selective in that they exhibited at least a 2:1 difference in the number of action potentials elicited by a spot moved in 2 opposing directions. Typically, cells were broadly tuned for this parameter, with reliable responses being evident at least $45^{\circ}$ to either side of the preferred direction of stimulus movement. Distributions of the preferred directions of these cells, defined as the direction that yielded the greatest difference between 2 opposing directions of movement, are illustrated in Figure 6. As may be seen, there is a clear overall bias for directions in the horizontal plane.

In Figure 7 we have replotted these data as a function of the position of the receptive field center with respect to the area centralis representation. This provided the axial directional preference, defined as the difference between the angles formed by the preferred direction vector with the horizontal and that formed by the horizontal with a line drawn between the receptive field center and the area centralis. As may be seen, there is no obvious bias in the axial direction preference in this sample of cells in that centrifugal, centripetal, and orthogonal directions are represented nearly equally.

\section{Orientation selectivity}

Almost half of the cells $(102 / 219)$ were found to be orientation selective, and the distribution of the preferred orientations of these neurons is illustrated in Figure 8. As a group, the oriented cells had significantly smaller receptive fields than the nonoriented cells ( $\left.120 \mathrm{vs} 169 \mathrm{deg}^{2}, t=2.72, d f=203, p=0.007\right)$. In all other respects the oriented cells did not differ appreciably

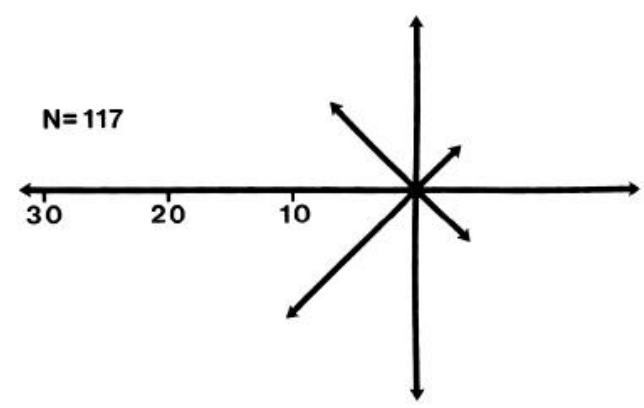

TEMPORAL

NASAL

Figure 6. The distribution of the preferred directions of 117 LPI cells. Nasal designates peripheral to central directional preferences along the horizontal visual axis of the contralateral hemifield. The length of each arrow is scaled to the number of cells preferring a given direction of stimulus movement.

from the overall population of neurons within the LPl. For instance, $55 \%$ of the oriented cells were also directionally selective, whereas, as indicated above, overall $58 \%$ of the LPI cells were directional.

In plotting receptive fields it became apparent that many of the LPI cells required fairly specific stimulus orientations to yield brisk discharges. This observation was quantified by obtaining orientation tuning curves for 48 neurons in the striate-recipient zone. An example of one such curve is depicted in Figure 9 (insert). In each case we determined the angle through which the stimulus had to be rotated to reduce the peak response by half. The distribution of these half-width at half-height measurements for the entire sample of cells is illustrated in Figure 9. The mean value for the overall sample was $27^{\circ}$, confirming our qualitative observations that these cells were rather tightly tuned for stimulus orientation.

\section{Receptive field organization}

We examined the internal organization of the receptive fields by flashing a small stimulus (spot or bar yielding reliable responses) at various positions across the activating region of the field. In all cases only phasic responses were obtained, and most cells responded to both stimulus onset and offset. However, response polarity often varied as a function of stimulus position within the receptive field. For 46 cells we constructed spatiotemporal maps of the receptive fields (see Fig. 10). In 32 of these neurons $(71 \%)$, the response pattern was dependent on stimulus position within the activating region. The majority $(63 \%)$ responded to stimulus onset as well as offset in the central region of the receptive field, while only "off" responses were evident at the edges of the field. In other cells $(21 \%)$, the central "onoff" region was flanked by edges that yielded only "on" responses. The remainder of the neurons $(16 \%)$ showed a bipartite receptive field organization: one region yielding "on-off" responses and an adjacent region with only "on" or "off" discharges.

We also tested for the presence of "silent" suppressive areas surrounding the activating region of the receptive field. In the majority of cells (99/152), flashed stimuli larger than the activating region of the receptive field produced either complete or partial response suppression. The proportion of cells exhibiting response suppression did not differ appreciably as a function of the internal organization of the receptive field. 


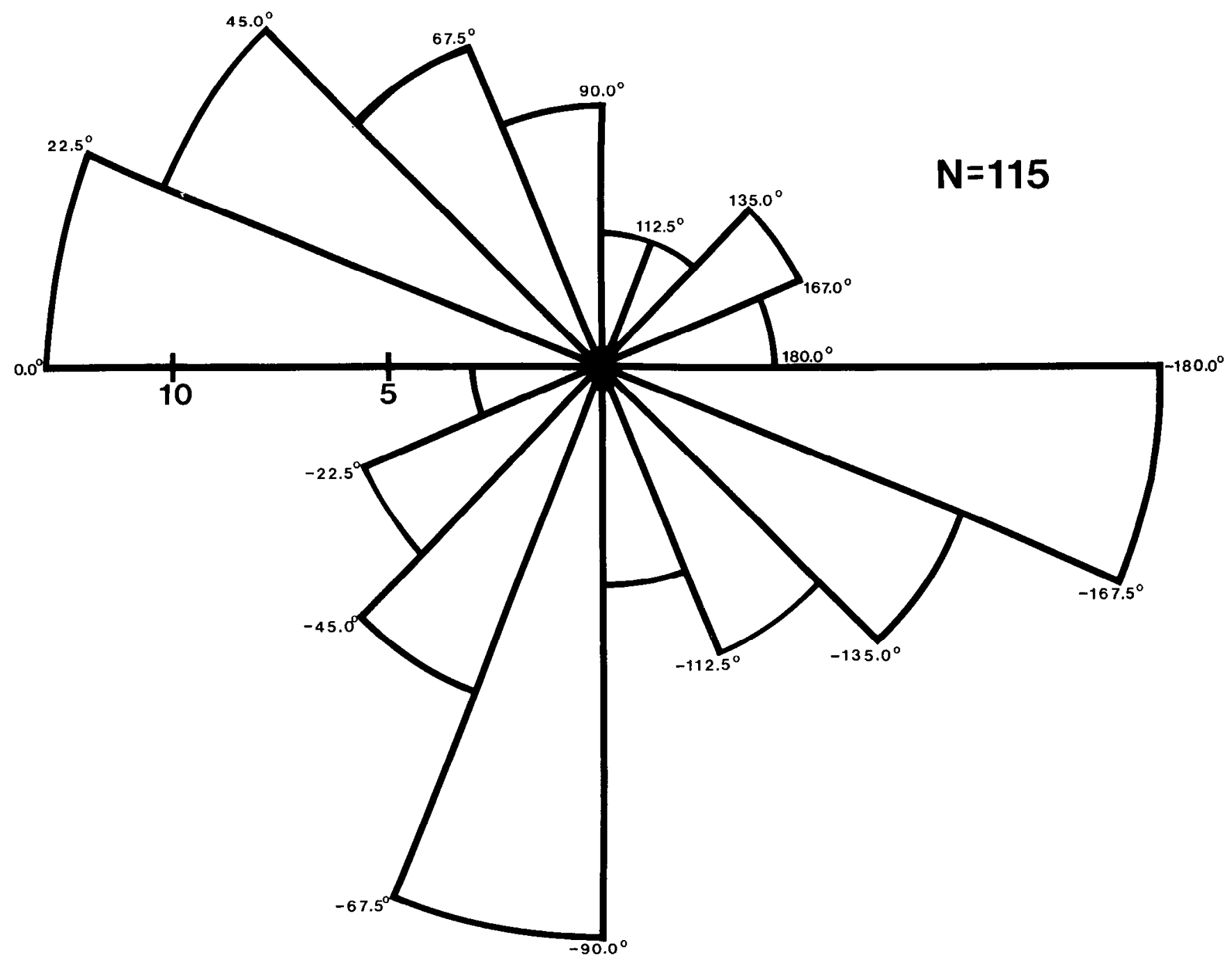

Figure 7. Distribution of axial direction preferences of $115 \mathrm{LPl}$ cells. The axial direction preference refers to the difference (in deg) between the angle formed by the preferred direction vector with the horizontal and the angle formed between the horizontal meridian and a line drawn between the receptive field center and the area centralis. Horizontal movements towards the vertical meridian were designated $0^{\circ}$ and those in the opposite direction $180^{\circ}$. The angles formed by the receptive field position are referenced to the $0^{\circ}$ (nasal) position.

\section{Functional organization in the dorsolateral segment of the LPI}

In the dorsolateral segment of the $\mathrm{LPl}$, the region adjacent to the pulvinar, a markedly different population of neurons was encountered. Of the 47 cells we isolated here, only $68 \%(n=$ 32) could be securely driven by the type of visual stimuli we employed in this study. In several instances, we tried less conventional methods such as textured patterns, rotating stripes, and even 3-dimensional displays of various portions of the experimenter's body; these attempts served mainly to prolong the recording session without increasing the number of visually responsive neurons.

The visual cells recorded in this segment had enormous receptive fields, averaging $518 \pm 452 \mathrm{deg}^{2}$, as compared with 160 $\pm 146 \mathrm{deg}^{2}$, at equivalent eccentricities, in the main segment of the LPI. Additionally, only $15 \%$ of the cells were orientation selective, and all of these were broadly tuned for this dimension. Directional selectivity was observed in $27 \%$ of the cells in this population. As in the main segment, most of the cells were binocular $(77 \%)$; however, the small number of neurons yielding reliable visual responses precluded a meaningful analysis of ocular dominance groupings. These data are summarized and contrasted with the response properties of cells in the main segment of the LPl in Table 1. We refer to the main segment as LPl-1 and the dorsolateral subarea as LPI-2.

As indicated previously, the 2 functionally distinct subregions of LPl are depicted in Figure 3. It should be noted, however, that our methods did not permit us to ascertain the precise boundary between the 2 subareas. There were no obvious morphological differences apparent to us in the Nissl material, and the histochemistry also revealed a relatively uniform staining pattern across the LPl. Typically, we isolated 6-10 cells per penetration, and because of this low sampling density, the electrophysiological data also did not provide an accurate index of the transition from one subarea to the other. Thus, Figure 3 provides only approximations of the extent of the 2 subareas in the LPl. Nevertheless, it is important to stress that functional differences between LPl-1 and LPl-2 were clearly evident in all penetrations, provided a sufficient number of cells were isolated in each subarea. 


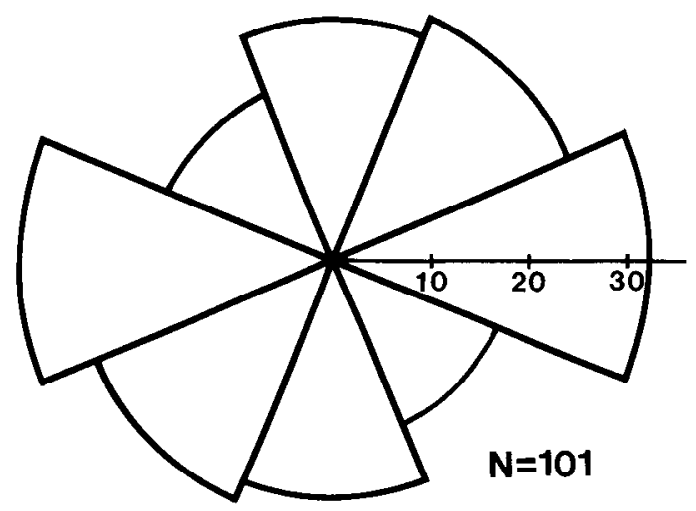

Figure 8. Distribution of the preferred orientations of 101 oriented cells isolated in the LPl. Each component of the figure is scaled to show the number of cells with preferred orientations within $\pm 45^{\circ}$ of a major axis (horizontal, vertical, or obliquc).

This point is documented in Figure 11, which illustrates rcconstructions of 3 different penetrations typical of the entire series. In each instance, the cells isolated in the dorsal portion of the penetration (i.e., in the subarea we designated the LPl-2) had very large receptive fields and a low degree of response specificity or were visually unresponsive. Also typical (as depicted in Fig. 11) was a segment of the track below this subarea in which we could not isolate cells. More ventral in the penetration we encountered cells (in LPI-1) that responded securely to visual stimuli with relatively small receptive fields and a high degree of response specificity.

\section{Discussion}

\section{Two striate-recipient subareas}

Our results indicate that the lateral division of the cat's LP nucleus may be apportioned into 2 subareas on the basis of cellular response properties. The main segment, which we designated the LPl-1, contains cells that respond reliably to the types of visual stimuli commonly employed by visual neurophysiologists. Most of these neurons have relatively small, welldefined receptive fields and show a substantial degree of response specificity. In contrast, in the small dorsomedial portion of the LPl, designated the LPI-2, many neurons are not visually responsive. The cells in LPI-2 that do respond to visual stimuli have huge receptive fields and generally show poor response specificity.

The foregoing observations raise 2 questions. Is LPl-2 a part of the striate-recipient zone and, if so, what might account for the differences in the functional organization of these 2 subregions of the LP1? Both of these questions can be addressed by considering the results of recent anatomical studies.

Focal electrophoretic deposits of WGA-HRP in the region corresponding to LPl-2 resulted in backfilled pyramidal cells

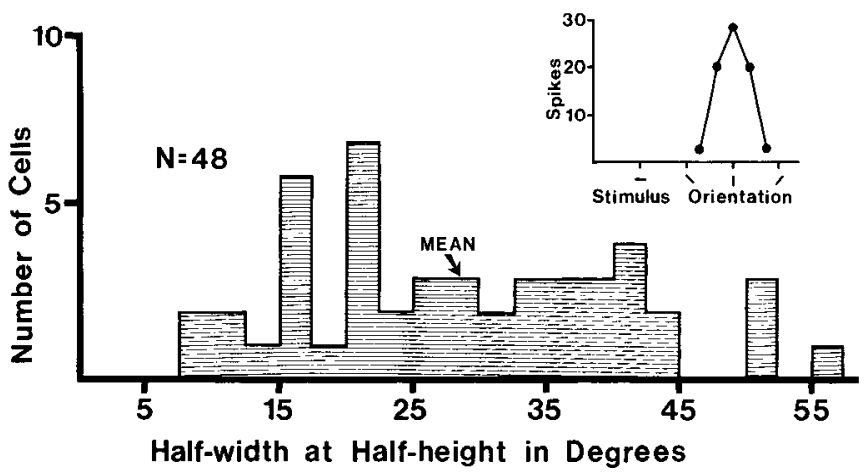

Figure 9. Quantitative measure of orientation selectivity for $48 \mathrm{LPl}$ cells. Measurements for each cell were taken as shown in the inset. (The number of spikes in response to a stationary bar, $1.5^{\circ}$ wide and of variable length, presented in the center of the receptive field was obtained on-line for 10 successive stimulus presentations. The angle of orientation was altered by $15^{\circ}$ and the procedure repeated.) The value for each cell is given as the angle in degrees through which the stimulus was rotated to reduce the response by half (half-width at half-height). The average value was $27^{\circ}$.

within layers 5 and 6 of areas 17 and 18 (Abramson and Chalupa, 1985a, see Figs. 5, 7). Thus, LPl-2 is a part of the striaterecipient region of the LP-pulvinar complex.

More recently, we showed that the region we have denoted as LPl-2 receives projections from cells in the stratum griseum superficiale of the superior colliculus, whereas LPl-1 does not receive collicular inputs (Abramson and Chalupa, 1988). Furthermore, the LPl-2 region also receives projections from the hypothalamus as well as the cerebellum (Yoshii et al., 1978; Rodrigo-Angulo and Reinoso-Suarez, 1984; Abramson and Chalupa 1985b). It seems reasonable to suggest that the diverse subcortical inputs to LPl-2 underlie the differences in response properties between the $2 \mathrm{LPl}$ regions. It is not known, however, if these 2 subareas contain 2 representations of the visual field. Such a dual representation has not been reported by prior electrophysiological mapping studies (Mason, 1981; Raczkowski and Rosenquist, 1981; Hutchins and Updyke, 1984). In the present study, the very large receptive fields and the presence of many cells in the LPl-2 that could not be visually activated precluded an assessment of retinotopy in this subarea.

It should be noted that an apportionment of the LP nucleus into a shell and core region was suggested earlier by Updyke (1983) on the basis of a detailed examination of Nissl material. His core region appears to correspond to the LPl-1, while the dorsal-shell region appears to be largely equivalent to the LPl-2 subarea.

\section{Comparison with the principal tectorecipient zone}

One of the objectives of the present study was to determine how the functional organization of the striate-recipient area differs

Table 1. Response properties of LPI neurons

\begin{tabular}{llllll} 
& $\begin{array}{l}\text { Average receptive } \\
\text { field size } \\
\left(\mathrm{deg}^{2}\right)\end{array}$ & \multicolumn{3}{l}{ Percent of cells } \\
\cline { 2 - 5 } & $\begin{array}{l}\text { Visually } \\
\text { responsive }\end{array}$ & $\begin{array}{l}\text { Orientation } \\
\text { selective }\end{array}$ & Directional & Binocular \\
\hline LPl-1 & $160 \pm 146(223)$ & $97(232)$ & $46(219)$ & $58(219)$ & $86(212)$ \\
LP1-2 & $518 \pm 452(32)$ & $68(47)$ & $15(32)$ & $27(32)$ & $77(30)$ \\
\hline
\end{tabular}

Values in parentheses indicate the number of cells tested. 

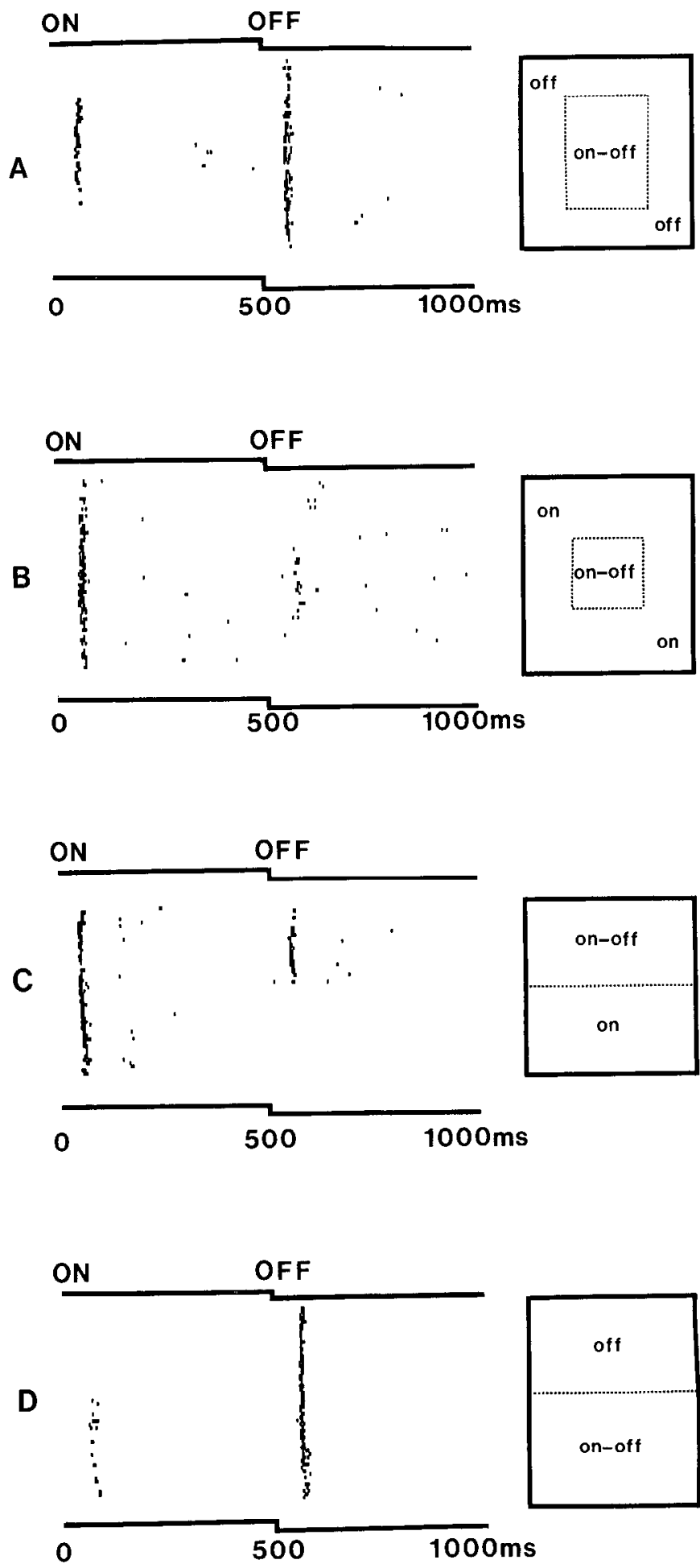

Figure 10. Receptive field organization for LPI cells. $A-D$, Spatialtemporal maps of the receptive fields of 4 cells. The stimulus was a flashing bar of light $\left(1^{\circ} \times 30^{\circ}\right)$ moved across the receptive field in 60 equally spaced steps. Time is represented along the horizontal axis and the normalized distance across the receptive field on the vertical axis. Each dot represents an action potential. The resolution of the raster was $4 \mathrm{msec}$. At right are schematic spatial maps showing the major response areas within the receptive field of each cell. $A$ and $B$, Central "on-off" area with either "off" $(A)$ or "on" $(B)$ surround. $C$ and $D$, Bipartite field with one "on-off" region and one "on" $(C)$ or "off" $(D)$ region. from that of the principal tectorecipient region in the medial LP (the LPm). For such a comparison we will rely upon our sample of LPl-1 cells and the results of a previous study from this laboratory, carried out under identical conditions, that dealt with the LPm (Chalupa et al., 1983).

Three main response characteristics clearly distinguish the visual cells in the striate and principal tectorecipient zones of the LP nucleus.

First, the average size of the receptive fields is substantially smaller in the striate-recipient zone than in the tectorecipient region. Across all eccentricities (from $0^{\circ}$ to $40^{\circ}$ ) the average receptive field area of $\mathrm{LPl}-1$ cells is one-fourth the size of the LPm cells (160 vs $645 \mathrm{deg}^{2}$, respectively).

Second, the degree of orientation selectivity is different in these 2 divisions of the LP-pulvinar complex. This is indicated by an incidence of orientation-selective cells that is almost twice that in the striate-recipient as in the principal tectorecipient $7.0 n e$ (49 vs $27 \%$ ) Furthermore, the orientation tuning of cells in the striate-recipient zone is quite precise, as indicated by a mean value of half-width at half-height of $27^{\circ}$. A quantitative analysis of this response property has not been carried out for LPm neurons, although virtually all oriented neurons in the tectorecipient zone respond well to bars of light more than $30^{\circ}$ from the preferred orientation (Chalupa et al., 1983).

Third, the internal organization of the receptive fields is dissimilar in the striate and tectorecipient zones. Within the LPm virtually all cells have a homogenous organization, in that the response polarity ("on," "off," or "on-off") to a flashing stimulus is the same across all regions of the receptive field. In contrast, most cells in the LPl yield different response patterns when the position of the stimulus in the receptivc ficld is varicd.

Other visual response properties, including the degree of binocularity, directional selectivity, and the presence of silent suppressive regions surrounding the activating region of the receptive field are common to cells in the LPl and LPm. Particularly striking is the fact that the distributions of preferred directions are virtually indistinguishable in these two zones of the LP (compare Fig. 6A with 7B of Chalupa et al., 1983).

\section{Relation to cortical inputs}

A second objective of this study was to determine how the response properties of cells in the striate-recipient zone relate to the visual physiology of the cortical neurons projecting to the LP nucleus. In areas 17 and 18 this projection is derived from pyramidal neurons situated in layer 5 (Abramson and Chalupa, 1985a). While the receptive field properties of this specific population of cortical neurons have not been determined, it is highly likely that these are complex cells with functional properties similar or even identical to those of corticotectal neurons (Palmer and Rosenquist, 1974; Harvey, 1980). Lund et al. (1979) have suggested that large pyramidal neurons in layer 5 of area 17 send axon collaterals to both the superior colliculus and the lateral posterior nucleus. These complex cells are strongly binocular, respond to very fast stimulus movements, are often directionally selective, and show orientation tuning curves very similar to those in our sample of LPl-1 neurons (Palmer and Rosenquist, 1974; Gilbert, 1977; Harvey, 1980). However, unlike complex cells in layer 5, most LPI cells have receptive fields with subregions that yield responses of different polarities. Such an organization could reflect the convergence of 2 or more complex cells onto a single LPI neuron.

Additional contributions to the functional properties of LPl 

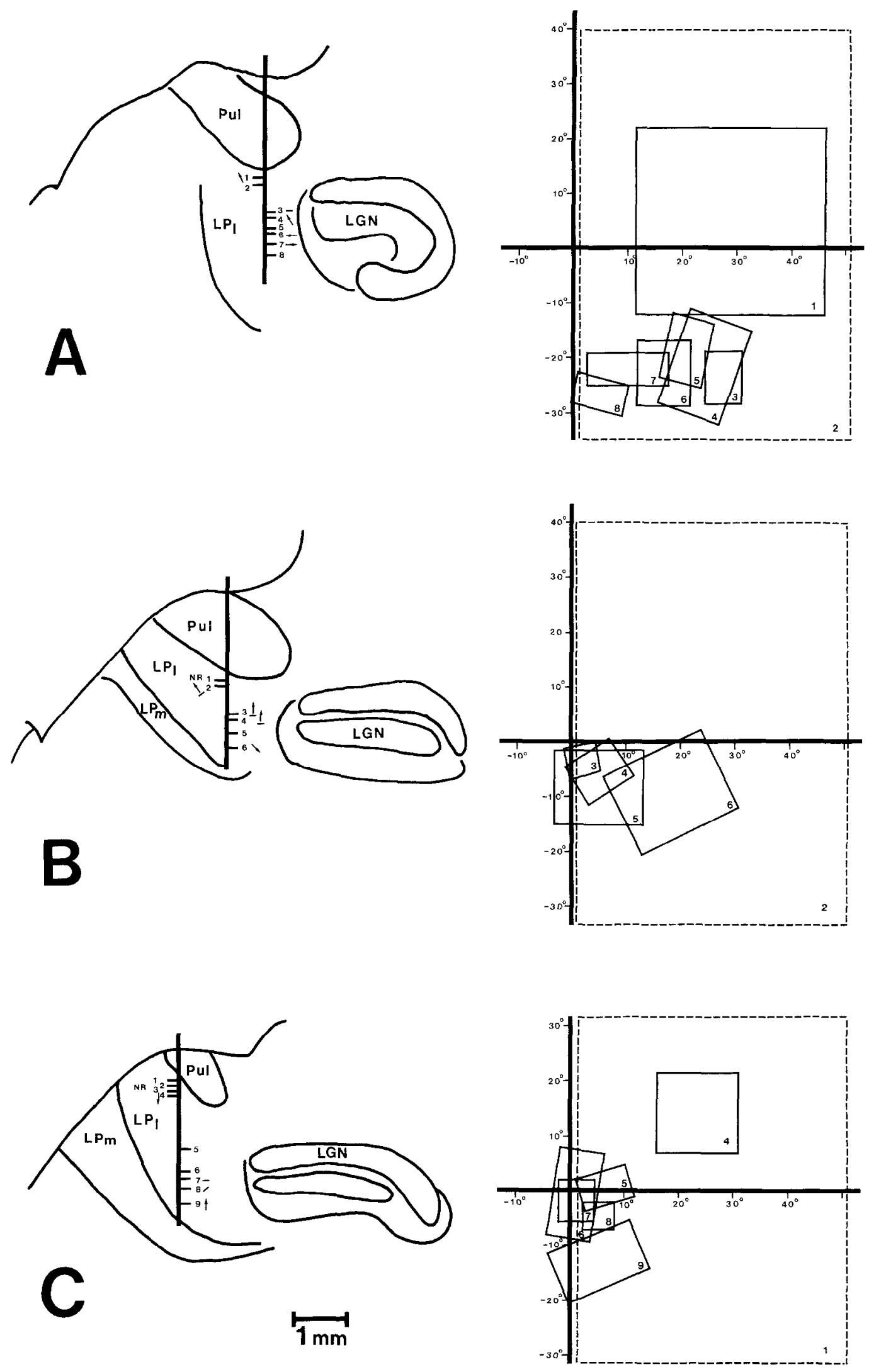

Figure 11. Reconstructions of 3 typical electrode penetrations through the LPl. Left, the location of cells along each electrode penetration is indicated by hatch marks on the line drawings of the thalamic sections. The section shown in $A$ was taken at a Horsley-Clarke frontal coordinate of about A7.5, B at A6.5, and $C$ at A6.0. Cells isolated within the LPl-1 are denoted by numbers to the right of the electrode tracks, while cells in the LPI-2 are to the left. The arrows and bars adjacent to the numbers represent the direction and orientation preferences of the cells, respectively. Cells unresponsive to visual stimuli ( 1 in $B$, and 2 and 3 in $C$ ) are denoted as NR. All other abbreviations are as in Figure 1 . Right, the location and size of receptive fields plotted for the visual cells in these 3 penetrations. The number in the lower right-hand corner of each receptive field refers to the corresponding numbered cell along the electrode track. Cells whose receptive fields are denoted by dashed lines responded to visual stimuli throughout the contralateral hemifield. 
cells may be provided by the extrastriate visual areas, particularly by cells in the visual areas of the lateral suprasylvian (LS) cortex. While a laminar analysis of receptive field properties in extrastriate cortex has not been carried out, it is well-documented that most LS neurons are directionally selective and prefer directions of stimulus movement along the horizontal meridian, away from the center of gaze (Hubel and Wiesel, 1969; Spear and Baumann, 1975; Camarda and Rizzolatti, 1976). More recently, a centrifugal bias has been demonstrated for these neurons (Rauschecker et al., 1987). The majority of cells in the striate-recipient zone are directionally selective as well, but in contrast to LS neurons, the sample of LPI cells we studied showed a bias for movement in either direction along the horizontal plane. A rather unusual response property that is apparent in the LS and LPI is binocular facilitation; the proportion of cells reported by Rauschecker et al. (1987) to exhibit this type of binocular interaction in LS cortcx is $60 \%$, on the order of what we observed in the LPl.

Collectively, the results of the present study and our previous work on the principal tectorecipient zone of LP provide support for 2 related viewpoints concerning the organization of the LP. pulvinar complex. There are clear functional differences among the visual areas of the LP-pulvinar, and, to a large degree, these differences can be accounted for by the diverse sources of visual input to the subdivisions of the LP-pulvinar. These observations suggest that each of the visual areas of the LP-pulvinar subserves a somewhat different role in the processing of visual and visually related information. Presumably this involves the integration of information from distinct sets of cortical and/or subcortical visual structures, but it remains for future studies to elucidate such functions.

\section{References}

Abramson, B. P., and L. M. Chalupa (1985a) The laminar distribution of cortical connections with the tecto- and cortico-recipient zones in the cat's lateral posterior nucleus. Neuroscience 15: 81-95.

Abramson, B. P., and L. M. Chalupa (1985b) Two subregions within the striate-recipient area of the cat's LP complex. Soc. Neurosci. Abstr. 11: 234 .

Abramson, B. P., and L. M. Chalupa (1988) The projections of the superior colliculus to the extrageniculate visual thalamus of the cat. J. Comp. Neurol. 271: 397-418.

Berman, N. (1977) Connections of the pretectum of the cat. J. Comp. Neurol. 174: 227-254.

Berson, D. M., and A. M. Graybiel (1983) Organization of the striaterecipient zone of the cat's lateralis posterior-pulvinar complex and its relations with the geniculostriate system. Neuroscience 9: 337 372.

Bishop, P. O., W. Kozak, and G. J. Vakkur (1962) Some quantitative aspects of the cat's eye: Axis and plane of reference, visual field coordinates and optics. J. Physiol. (Lond.) 162: 466-502.

Butcher, L. L., S. M. Eastgate, and G. K. Hodge (1974) Evidence that punctate intracerebral administration of 6-hydoxydopamine fails to produce selective neuronal degeneration: Comparison with copper sulfate and factors governing the deportment of fluids injected into the brain. Naunyn Schmiedbergs Arch. Pharmacol. 285: 31-70.

Caldwell, R. B., and R. R. Mize (1981) Superior colliculus neurons which project to the cat lateral posterior complex have varying morphologies. J. Comp. Neurol. 203: 53-66.

Camarda, R., and G. Rizzolatti (1976) Visual receptive fields in the lateral suprasylvian area (Clare-Bishop area) of the cat. Brain Res. 101: $427-443$.

Chalupa, L. M. (1977) A review of cat and monkey studies implicating the pulvinar in visual function. Behav. Biol. 20: 149-167.

Chalupa, L. M., and S. E. Fish (1978) Response characteristics of visual and extra-visual neurons in the pulvinar and lateral posterior nuclei of the cat. Exp. Neurol. 36: 96-120.
Chalupa, L. M., R. W. Williams, and M. J. Hughes (1983) Visual response properties in the tecto-recipient zone of the cat's lateral posterior-pulvinar complex: A comparison with the superior colliculus. J. Neurosci. 3: 2587-2596.

Fish, S. E., and L. M. Chalupa (1979) Functional properties of pulvinar-lateral posterior neurons which receive input from the superior colliculus. Exp. Brain Res. 36: 245-257.

Gilbert, C. D. (1977) Laminar differences in the receptive field properties of cells in cat primary visual cortex. J. Physiol. (Lond.) 268: $391-421$.

Godfraind, J. M., M. Meulders, and C. Veraart (1972) Visual properties of neurons in pulvinar, nucleus lateralis posterior and nucleus suprageniculatus thalami of the cat. I. Qualitative investigation. Brain Res. 44: 503-526.

Graham, J. (1977) An autoradiographic study of the efferent connections of the superior colliculus of the cat. J. Comp. Neurol. 173:629654.

Graybiel, A. M., and D. M. Berson (1980) Histochemical identification and afferent connections of subdivisions in the lateralis posteriorpulvinar complex and related thalamic nuclei in the cat. Neuroscience 5: $1175-1238$.

Harvey, A. R. (1980) A physiological analysis of subcortical and commissural projections of areas 17 and 18 of the cat. J. Physiol. (Lond.) 302: 507-534.

Hubel, D. H., and T. N. Wiesel (1962) Receptive fields, binocular interaction and functional architecture in the cat's visual cortex. $J$. Physiol. (Lond.) 160: 106-154.

Hubel, D. H., and T. N. Wiesel (1969) Visual area of the lateral suprasylvian gyrus (Clare-Bishop area) of the cat. J. Physiol. (Lond.) 202: 251-260.

Hutchins, B., and B. V. Updyke (1984) Retinotopic organization within the lateral posterior complex of the cat. Soc. Neurosci. Abstr. 10: 727.

Itoh, K. (1977) Efferent projections of the pretectum of the cat. Exp. Brain Res. 30: 89-105.

Karnovsky, M. J., and L. Roots (1964) A direct coloring thiocholine method for cholinesterases. J. Histochem. Cytochem. 12: 219-221.

Kawamura, S. (1974) Topical organization of the extrastriate visual system in the cat. Exp. Neurol. 45: 451-461.

Kawamura, S., and E. Kobayashi (1975) Identification of some tectothalamic fibers in the cat. Brain Res. 91: 281-285.

Kawamura, S., J. M. Sprague, and K. Niimi (1974) Corticofugal projections from the visual cortices to the thalamus, pretectum and superior colliculus in the cat. J. Comp. Neurol. 158: 339-362.

Lund, J. S., G. H. Henry, C. L. Macqueen, and A. R. Harvey (1979) Anatomical organization of the primary visual cortex, area 17, of the cat. A comparison with area 17 of the macaque monkey. J. Comp. Neurol. 184: 599-618.

Mason, R. (1978) Functional organization of the cat's pulvinar complex. Exp. Brain Res. 31: 51-66.

Mason, R. (1981) Differential responsiveness of cells in the visual zones of the cat's LP-pulvinar complex to visual stimuli. Exp. Brain Res. 43: 25-33.

Palmer, L. A., and A. C. Rosenquist (1974) Visual receptive fields of single striate cortical units projecting to the superior colliculus in the cat. Brain Res. 67: 27-42.

Raczkowski, D., and A. C. Rosenquist (1981) Retinotopic organization in the cat lateral posterior-pulvinar complex. Brain Res. 221: 185-191.

Raczkowski, D., and A. C. Rosenquist (1983) Connections of the multiple visual cortical areas with the lateral posterior-pulvinar complex in the cat. J. Neurosci. 3: 1912-1914.

Rauschecker, J. P., M. W. von Grunau, and C. Poulin (1987) Centrifugal organization of direction preference in the cat's lateral suprasylvian visual cortex and its relation to flow field processing. J. Neurosci. 7: 943-958.

Rodrigo-Angulo, M. L., and F. Reinoso-Suarez (1984) Cerebellar projections to the lateral posterior-pulvinar thalamic complex in the cat. Brain Res. 322: 172-176.

Spear, P. D., and T. D. Baumann (1975) Receptive-field characteristics of single ncurons in the latcral suprasylvian arca of the cat. J. Ncurophysiol. 38: 1403-1420.

Symonds, L. L., A. C. Rosenquist, S. B. Edwards, and L. A. Palmer (1981) Projections of the pulvinar-lateral posterior complex to visual cortical areas in the cat. Neuroscience 6: 1995-2020. 
Updyke, B. V. (1977) Topographic organization of the projections from cortical areas 17,18 , and 19 onto the thalamus, pretectum, and the superior colliculus of the cat. J. Comp. Neurol. 173: 81-121.

Updyke, B. V. (1981) Projections from visual areas of the middle suprasylvian sulcus onto the lateral posterior complex and adjacent thalamic nuclei in cat. J. Comp. Neurol. 201: 477-506.

Updyke, B. V. (1983) A re-evaluation of the functional organization and cytoarchitecture of the feline lateral posterior complex, with observations of adjoining cell groups. J. Comp. Neurol. 219: 143-181.
Weber, J. T., I.-L. Chen, and B. Hutchins (1986) The pretectal complex of the cat: Cells of origin of the projection to the pulvinar nucleus. Brain Res. 397: 389-394.

Yoshii, N., M. Fujii, and T. Mizokami (1978) Hypothalamic projections to the pulvinar-LP complex in the cat: A study by the HRP method. Brain Res. 155: 343-346. 